Hans-Lothar Merten

In Luxus investieren 
Hans-Lothar Merten

\section{In Luxus investieren}

Wie Anleger vom Konsumrausch der Reichen profitieren

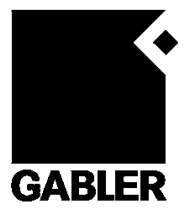


Bibliografische Information der Deutschen Nationalbibliothek

Die Deutsche Nationalbibliothek verzeichnet diese Publikation in der

Deutschen Nationalbibliografie; detaillierte bibliografische Daten sind im Internet über <http://dnb.d-nb.de> abrufbar.

Hans-Lothar Merten, Bankkaufmann und Betriebswirt, ist freier Publizist mit den Schwerpunkten Finanzen und Steuern.

1. Auflage 2009

Alle Rechte vorbehalten

(C) Gabler I GWV Fachverlage GmbH, Wiesbaden 2009

Lektorat: Stefanie Brich

Gabler ist Teil der Fachverlagsgruppe Springer Science+Business Media. www.gabler.de

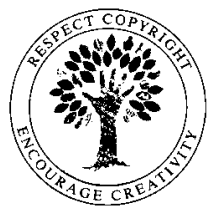

Das Werk einschließlich aller seiner Teile ist urheberrechtlich geschützt. Jede Verwertung außerhalb der engen Grenzen des Urheberrechtsgesetzes ist ohne Zustimmung des Verlags unzulässig und strafbar. Das gilt insbesondere für Vervielfältigungen, Übersetzungen, Mikroverfilmungen und die Einspeicherung und Verarbeitung in elektronischen Systemen.

Die Wiedergabe von Gebrauchsnamen, Handelsnamen, Warenbezeichnungen usw. in diesem Werk berechtigt auch ohne besondere Kennzeichnung nicht zu der Annahme, dass solche Namen im Sinne der Warenzeichen- und Markenschutz-Gesetzgebung als frei zu betrachten wären und daher von jedermann benutzt werden dürften.

Umschlaggestaltung: Nina Faber de.sign, Wiesbaden

Druck und buchbinderische Verarbeitung: Krips b.v., Meppel

Gedruckt auf säurefreiem und chlorfrei gebleichtem Papier

Printed in the Netherlands

ISBN 978-3-8349-0944-2 
Die Welt der Luxusgüter öffnet sich zunehmend auch für Privatanleger. In den wenigsten Fällen gilt diese Feststellung für die schönen Dinge des Lebens selbst, sondern für die Anlagemöglichkeiten in den edlen Konsumbereich. Zu den Aktien der Luxushersteller und einigen Fonds gesellt sich eine Reihe von Zertifikaten, mit denen Investoren an der Wachstumsphantasie der Branche teilhaben können. Banken beschreiben die Aussichten der Hersteller von Haute Couture, Schmuck oder Accessoires in den schillerndsten Farben, gehört doch auch die Oberschicht in aufstrebenden, wachstumsstarken Ländern wie China oder Russland zu den neuen Luxuskonsumenten. Rund 250 bis 300 Milliarden Jahresumsatz erwartet die Luxusbranche in den kommenden Jahren.

Abbildung 1: Wer am meisten für Luxusgüter ausgibt nach Herkunft der Käufer, Anteil am Weltmarkt in Prozent

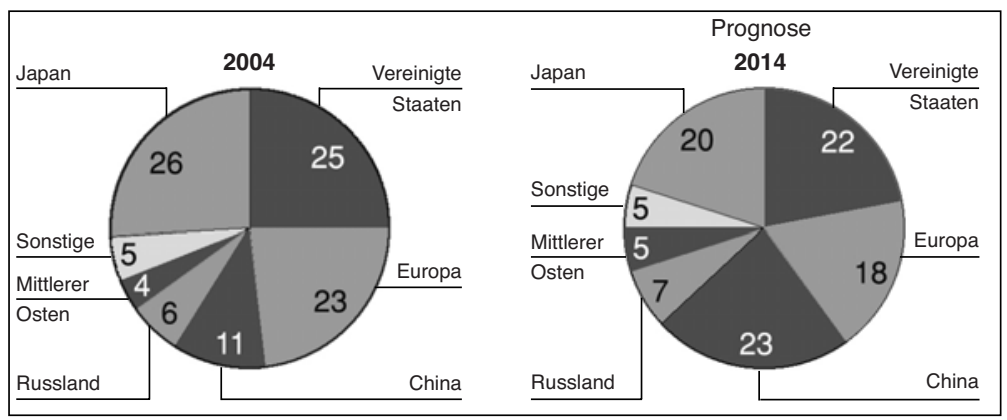

Quelle: Merrill Lynch, Vontobel Research

Potenzial ist vorhanden, denn die Zahl der vermögenden Privatpersonen nimmt zu. Aktuell verfügen weltweit über zehn Millionen Familien über ein jährliches Einkommen von mehr als einer Million USD. 2010 sollen es nach einer Analyse der Investmentbank Merrill Lynch bereits mehr als 15 Millionen Familien sein. Tendenz steigend. Vor allem in Russland, in den Ländern am Arabischen Golf, in China, Indien und Südostasien nimmt die Zahl der Millionäre und Milliardäre sprunghaft zu. Der neue Reichtum hat einen Run auf Luxusgüter aller Art ausgelöst. Ob Champagner, teure Uhren oder Juwelen die immer größere werdende Gruppe von Millionären zeigt ihren Reichtum gerne und setzt auf bekannte Marken. 
Die Macht der Marken schafft hohe Wettbewerbsbarrieren. Die Unternehmen profitieren weltweit von ihrem Bekanntheitsgrad. Es ist also völlig gleichgültig, in welchem Land der Erde die Nachfrage nach einem bestimmten Luxusgut besteht - Luxus hat immer Konjunktur. Das Luxussegment gilt als aussichtsreicher, konjunkturresistenter Wachstumsmarkt, der seit Jahren zweistellige Wachstumsraten verzeichnet. Und so übertrumpfen viele Luxusaktien seit längerem den breiten Markt. Dies hat sich auch in ihren Bewertungen niedergeschlagen. Mit einem Kurs-Gewinn-Verhältnis (KGV) von 18 auf Basis der für 2007 erwirtschafteten Gewinne je Aktie liegen die Luxustitel über dem Wert des Gesamtmarktes. Zum Vergleich: im Euro-Stoxx-50 liegt diese Bewertungskennzahl derzeit bei 11,5.

Als Wachstumstreiber des Luxussegments gelten der demografische Wandel sowie die Zunahme wohlhabender Bevölkerungsschichten vor allem in Schwellenländern. Mit der Alterung der Bevölkerung in den Industriestaaten und dem wachsenden Wohlstand steigt Analystenprognosen zufolge hier auch die Nachfrage nach Luxusgütern. Banken gründen ihre enormen Wachstumsausblicke in Bezug auf diese Branche jedoch vor allem auf die schnell wachsenden Schwellenländer. Bereits heute findet jeder dritte Luxusartikel den Weg zu Nationen wie China, Indien und Russland. Mit 12 Prozent des Gesamtumsatzes weltweit ist jetzt alleine China der drittgrößte Verbrauchermarkt von Luxuswaren. Vor fünf Jahren hatte der Anteil noch bei einem Prozent gelegen.

Spricht man aus anlagetechnischer Sicht von der Luxusgüterindustrie, fokussiert sich das auf börsennotierte Unternehmen, die primär Uhren, Schmuck und Mode herstellen und weltweit vermarkten - aufgelockert durch den Namen Porsche. Die LVMH-Gruppe oder etwa die Richemont Holding stehen mit Marken wie Louis Vuitton, Moet, Taittinger, Cartier, Dior, Montblanc oder IWC für Milliardenumsätze. Aber auch andere börsennotierte Unternehmen profitieren vom Kaufrausch, etwa der Seereisenveranstalter Carnival, der Motorradhersteller Harley Davidson, der französische Yachthersteller Rodriguez oder der dänische Unterhaltungselektronikanbieter Bang \& Olufson. Bei allen stehen die Zeichen für die nächsten Jahre auf Expansion. Dabei hat die Luxusgüterindustrie den Vorteil, dass sie nicht mit „spitzem Bleistift" rechnen muss. Je höher der Bekanntheitsgrad von Brands ist, umso leichter lassen sich am Markt auch teilweise exorbitante Preise durchsetzen. Das schlägt natürlich positiv auf die Gewinnentwicklung der Unternehmen durch. Hier bieten sich vor allem langfristig orientierten Anlegern attraktive Chancen.

Natürlich haben Konjunktureinbrüche, Aktiencrashs oder Ereignisse, die die Menschen stark verunsichern, Einfluss auf die Geschäftsentwicklung der Luxusgüterhersteller. Die Historie zeigt aber, dass sich die Branche von solchen Schocks sehr schnell wieder erholt. Das wird auch auf die sich seit Mitte 2007 abzeichnende konjunkturelle Eintrübung zutreffen. Diese wird nicht von langer Dauer sein.

Welches Potenzial bieten also die klassischen Märkte in Europa und Nordamerika für Luxusgüterhersteller? Hält die hohe Nachfrage nach Luxusprodukten aus den Golfstaaten, Russland, China und Indien an? Und wie viel "Luxus" im Depot darf es sein? Wir sind den Fragen nachgegangen.

H.-Lothar Merten 


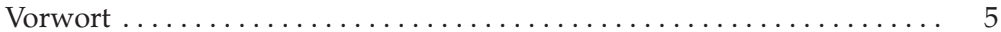

$1 \quad$ Was ist Luxus? . . . . . . . . . . . . . . . . . . . . . 13

2 Millionäre haben Konjunktur - sie investieren in Luxus und

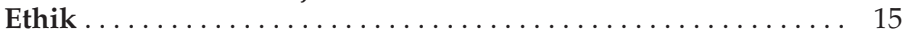

$2.1 \quad$ Investments für den guten Zweck $\ldots \ldots \ldots \ldots \ldots \ldots \ldots \ldots \ldots$

2.2 Was Anlage-Strategen Superreichen bei der Vermögensanlage raten

$3 \quad$ Die Reichsten der Reichen $\ldots \ldots \ldots \ldots \ldots \ldots \ldots \ldots$

$4 \quad$ Reichtum - Reiche und Vermögende ............... 25

$5 \quad$ Reiche investieren besser $\ldots \ldots \ldots \ldots \ldots \ldots \ldots \ldots \ldots \ldots$

$6 \quad$ Was macht Luxus aus? . . . . . . . . . . . . . . . . 31

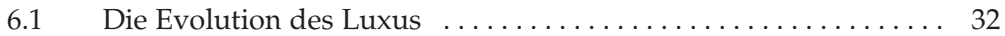

6.2 Die Luxus-Trends der Zukunft ................. 33

6.3 Wie wirkt sich die Entwicklung auf Luxusmarken aus? . . . . . . . 34

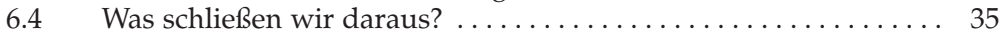

$7 \quad$ Luxusartikel sind en vogue $\ldots \ldots \ldots \ldots \ldots \ldots \ldots \ldots \ldots \ldots$

7.1 Wer schürt die Nachfrage nach Labels wie Cartier, Hermès oder

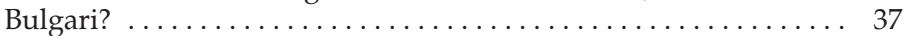

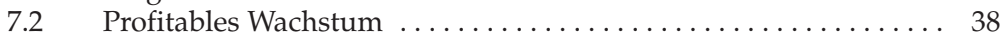

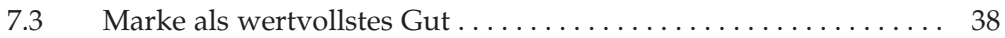

7.4 Investieren in Luxus - Chancen und Risiken . . . . . . . . . . . . . 39

$8 \quad$ Der weltweite Luxusgütermarkt $\ldots \ldots \ldots \ldots \ldots \ldots \ldots \ldots$

8.1 Die populärsten Luxusprodukte für Männer . . . . . . . . . . 46

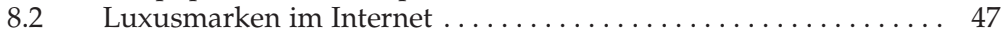

$8.3 \quad$ Luxusgüterhersteller im Aufwind .................... 48

$8.4 \quad$ Luxus - ein werthaltiges Investment - Fakten . . . . . . . . . . . . 49

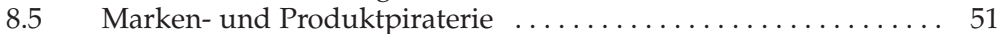

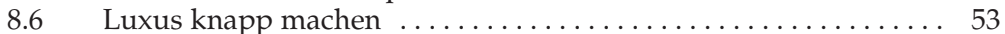

8.7 Die wertvollsten Marken der Welt . . . . . . . . . . . . . . . . 54

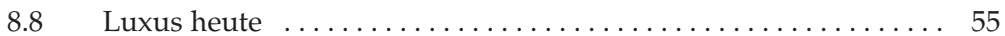


$9 \quad$ In den Luxusgüterbereich investieren $\ldots \ldots \ldots \ldots \ldots \ldots \ldots$

9.1 Wer reich werden will, sollte von den Reichen lernen . . . . . . 57

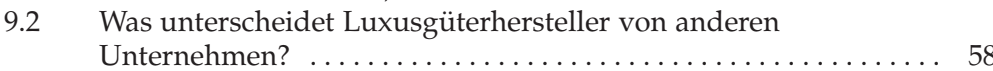

9.3 Gegenwärtige Situation und Ausblick ................ 59

9.4 Auch Finanzinvestoren entdecken den Luxus ............. 60

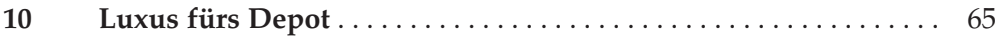

11 Ausgewählte Investments in Luxus ................ 69

11.1 Mit Gold \& Platin gegen Krisen \& Inflation ... . . . . . . . . . . . 69

11.1.1 Wie können Investoren von dieser Entwicklung profitieren? . . . 72

11.1.2 Gold \& Platin - Versicherung gegen Inflation $\ldots \ldots \ldots \ldots \ldots \ldots 73$

11.1.3 Wissenswertes rund um Gold \& Platin ............... 74

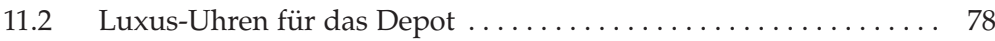

11.3 Diamanten - unvergänglich, aber nicht für alle Ewigkeit . . . . . 85

11.4 Auslandsimmobilie - Luxus pur . . . . . . . . . . . . . . 91

11.4.1 Paradiesisch investieren - die eigene Insel $\ldots \ldots \ldots \ldots \ldots \ldots . \ldots 5$

11.4.2 Auslands-Immobilien als Kapitalanlage ................. 96

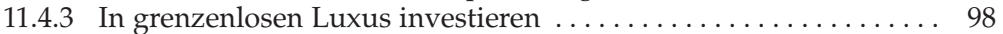

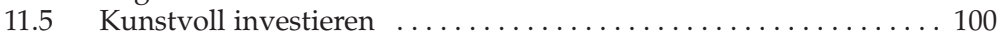

11.5.1 Kunstmarktsektoren ............................ 100

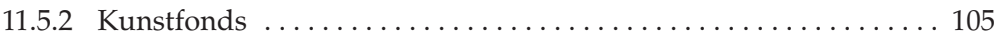

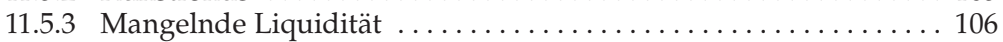

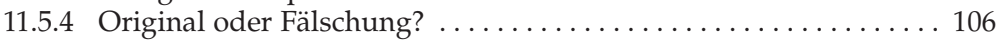

11.5.5 Von Rekord zu Rekord . . . . . . . . . . . . . . . . . . . 107

11.5.6 Gute Geschäfte mit Fotografie . . . . . . . . . . . . . . . . . . . 109

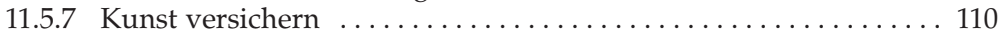

11.6 Rendite vom Rebstock . . . . . . . . . . . . . . . . . . . . 110

11.7 Mobile Wertanlage mit Genuss . . . . . . . . . . . . . . . 114

11.7.1 Classic Cars: eine Definition $\ldots \ldots \ldots \ldots \ldots \ldots \ldots \ldots \ldots \ldots \ldots \ldots$

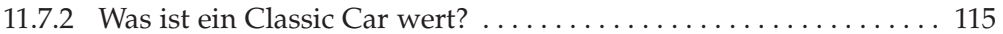

11.7.3 Luxusautos auf dem Weg zum Öko-Luxus . . . . . . . . . . . . . 117

11.7.4 The Gentleman's Sportcar ... . . . . . . . . . . . . . . . . . 117

11.8 Meissener Porzellan . . . . . . . . . . . . . . . . . . . . . . . . . . 118

11.9 Münzen als Kapitalanlage . . . . . . . . . . . . . . . . . . . . . . 119

11.10 Kunst - Luxus-Stoff für Prestige und Masse . . . . . . . . . . . . 120

11.11 Luxus auf Pump . . . . . . . . . . . . . . . . . . 121

11.12 Das Luxussegment ......................... 121

12 Luxusunternehmen auf dem Prüfstand $\ldots \ldots \ldots \ldots \ldots \ldots \ldots$

12.1 Besonderheiten der Luxusgüter . . . . . . . . . . . . . . . 123

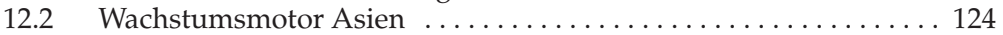

12.3 Rekordzahlen für 2007 und $2008 \ldots \ldots \ldots \ldots \ldots \ldots \ldots \ldots \ldots \ldots \ldots$

12.4 Die Subprime-Krise hinterlässt Spuren . . . . . . . . . . . . . . . 125

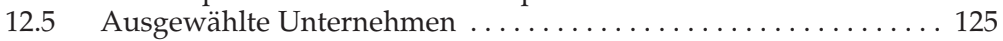

$13 \quad$ Luxusinvestments leicht gemacht $\ldots \ldots \ldots \ldots \ldots \ldots \ldots \ldots$ 
14 Der andere Luxus - Ethikfonds . . . . . . . . . . . . . . . 141

14.1 Ethik-Trends im "globalen Dorf" .................. 141

14.2 Performance-Pep mit extrafinanziellen Investmentkriterien . . . . 143

14.3 Themenfonds: Ökologie/Ethik/Nachhaltigkeit . . . . . . . . . . . . 145

$15 \quad$ Verliert der Luxus seinen Glanz? . . . . . . . . . . . . . . . . . . . 151

Institutionen - Personen - Unternehmen $\ldots \ldots \ldots \ldots \ldots \ldots \ldots \ldots \ldots$

Quellen ............................................. 159 
„Luxus muss sein.

Wenn die Reichen nichts verschwenden, verhungern die Armen."

Voltaire 